\title{
Welche Lungenkranke gehören in die Heilstätten?
}

Eine Berichtigung zu Köhlers „Kritische Nachlese zur IV. Tuberkuloseärzteversammlung in Berlin“ (cf. d. Zeitschr. Bd. VIII, H. 1).

Von

Dr. med. G. Schröder, Schömberg.

In seinem Berichte ist F. Köhler bei der Besprechung meines Berliner Referates "über die Dauer der Heilstättenkuren“ ein Irrtum untergelaufen, der im Interesse der Sache berichtigt werden muss. Um zu einem möglichst einwandfreien Urteil über die Kurdauer in den Heilstätten zu kommen, musste ich untersuchen, welche Fälle von chronischer Lungentuberkulose der Heilstättenbehandlung bedürftig sind. Ich kam zu dem Schluss, dass nur aktive Formen der Phthise in die Heilstätten gehören und dassdieAktivität des Prozesses vor der Hand noch in erster Linie durch eine exakte Anamnese und klinische Beobachtung unter Würdigung aller Symptome festgestellt werden kann.

Den aktiven Fällen stellte ich die latenten und stationären Formen gegenüber, d. h. solche, bei denen die Tuberkulose sich chronisch latent in den Drüsen abspielt oder die Untersuchung in den Lungen nur Residuen früherer aktiver Prozesse nachweist, die keine Krankheitszeichen mehr machen; ich meine: Pleuraschwarten und Spitzenschrumpfungen. Es schliessen sich hier noch sekundäre Emphysembildung, chronische Bronchitiden mit eventueller Bronchiektasie an. Diese latenten und stationären Formen können sehr gut und billiger Kuren in Walderholungsstätten und offenen Kurorten durchmachen; sie kann man auch zur Beobachtung, falls sie noch keine Anstaltsbehandlung genossen, in Dispensaires, Polikliniken ambulant behandeln. Die vielleicht leicht reduzierte Arbeits- 
fähigkeit solcher Kranker lässt sich überraschend schnell in wenigen Wochen steigern oder sogar wiederherstellen. Eine eventuell positive probatorische Tuberkulininjektion kann bei ihnen nicht zur Aufnahme in eine Heilstätte und Absolvierung einer monatelangen strengen Anstaltskur die Entscheidung abgeben. - Niemals unterschied ich zwischen latenten und of fenen Tuberkulosen. Hier hat Köhler mich missverstanden. Es würde von meiner Sejte eine grosse Rückständigkeit vorliegen, wenn ich nur offene Tuberkulosen, d. h. Bazillenhuster in die Anstalten aufgenommen sehen wollte.

In der Einleitung meines Referates sagte ich ausdrücklich, dass selbstverständlich die beginnende Lungentuberkulose, also das Anfangsstadium in die Anstalten gehört. Hier fehlen meist die Bazillen noch. Es fehlt häufig der Auswurf. - Ich verlange aber, dass die in den Anstalten Aufgenommenen des I. Stadiums wirklich krank und arbeitsunfähig sind, nicht etwa nur auf Tuberkulin positiv reagieren. Das Anfangsstadium der noch geschlossenen aktiven Tuberkulose macht stets genügende, oft sogar schwere Symptome und bedingt Arbeitsunfäbigkeit, das Latenzstadium der Tuberkulose pflegt in der Regel keine Erscheinungen zu machen. Es muss also geschieden werden: alstive und latente Formen, nicht etwa offene und latente, wie Köhler es tut. - Hält man die latenten Fälle und chronischstationären Phthisen von den Heilstätten fern, und versucht es, in den freigewordenen Plätzen auch schwerer Kranke mit Fieber und Larynxtuberkulose aufzunehmen, bei denen durch eine Vorbeobachtung die Prognose nicht ungünstig gestellt ist, so wird das Ansehen der Heilstätten bei den Ärzten steigen; wir werden in ihnen stets wirkliche Heilanstalten für Kranke sehen. 